\title{
The role of m6A RNA methylation-related IncRNAs in the prognosis and tumor immune microenvironment of papillary thyroid carcinoma
}

\section{WenLong Wang}

Xiangya Hospital Central South University https://orcid.org/0000-0003-4441-6027

\section{Cong Shen}

Xiangya Hospital Central South University

\section{Yunzhe Zhao}

Xiangya Hospital Central South University

\section{Botao Sun}

Xiangya Hospital Central South University

\section{Xiangyuan Qiu}

Xiangya Hospital Central South University

\section{Shujuan Yin}

Xiangya Hospital Central South University

Xinying Li ( $\square$ lixinyingcn@126.com )

Xiangya Hospital Central South University

\section{Research article}

Keywords: papillary thyroid carcinoma (PTC), tumor microenvironment, prognosis, N6-methylandenosine $(\mathrm{m} 6 \mathrm{~A})$

Posted Date: September 30th, 2021

DOI: https://doi.org/10.21203/rs.3.rs-903297/v1

License: (c) (1) This work is licensed under a Creative Commons Attribution 4.0 International License. Read Full License

Version of Record: A version of this preprint was published at Frontiers in Cell and Developmental Biology on January 3rd, 2022. See the published version at https://doi.org/10.3389/fcell.2021.719820. 
The role of m6A RNA methylation-related IncRNAs in the prognosis and tumor immune microenvironment of papillary thyroid carcinoma

\section{Wenlong Wang ${ }^{1,2}$, Chong Shen', Yunzhe Zhao', Botao Sun ${ }^{1}$, Xiangyuan Qiu', Shujuan Yin ${ }^{1}$, Xinying $\mathrm{Li}^{1,2 *}$.}

${ }^{1}$ Thyroid Surgery Department, Xiangya Hospital, Central South University, Changsha, 410008, China.

${ }^{2}$ National Clinical Research Center for Geriatric Disorders, Xiangya Hospital, Central South University, Changsha, 410008, Hunan Province, China.

\section{* Correspondence:}

Xinying Li

lixinyingen@126.com

\section{Abstract}

Background: Emerging evidence has indicated that N6-methylandenosine (m6A) RNA methylation plays a critical role in cancer development. However, the function of m6A RNA methylation-related long noncoding RNAs (m6A-lncRNAs) in papillary thyroid carcinoma (PTC) has never been reported. This study aimed to investigate the role of m6A-lncRNAs in the prognosis and tumor immune microenvironment of PTC. Methods: The gene expression data of lncRNAs and $20 \mathrm{~m} 6 \mathrm{~A}$ methylation regulators with corresponding clinicopathological information download from the Cancer Genome Atlas database. Based on consensus clustering analysis, LASSO Cox regression, nivariate and multivariate Cox regression analysis were used to determine the role of m6A-lncRNA in the prognosis and tumor immune microenvironment of PTC.

Results: Three subgroups (clusters 1,2, and 3) were identified by consensus clustering

of 19 prognosis-related m6A-lncRNA regulators, of which cluster 1 preferentially related with unfavorable prognosis, lower immune scores, and distinct immune infiltrate level. A risk-score model was established based on 8 prognosis-related m6AlncRNAs. Patients with a high-risk score had a worse prognosis and the ROC indicated a reliable prediction performance for patients with PTC (AUC $=0.802)$. As expected, the immune scores, infiltration levels of immune cells and ESTIMATE scores in the lowrisk subgroups were notably higher $(p<0.001)$ compared with those of high-risk subgroups. Furthermore, GSEA analysis showed that tumor associated pathways, hallmarks, and biological processes were remarkably enriched in the high-risk subgroup. Further analysis indicated that the risk score and age were independent prognostic factors for PTC. An integrated nomogram was constructed that accurately predicted the survival status (AUC $=0.963$ ). Moreover, a lncRNA-miRNA-mRNA regulated network was established based on seven prognosis-related m6A-lncRNAs. Additional, 30 clinical samples and different PTC cells were validated. 
Conclusions: This is the first study to reveal that m6A-lncRNAs play a vital role in the prognosis and TME of PTC. To a certain degree, m6A-lncRNAs can be considered as new, promising prognostic biomarkers and treatment targets.

Keywords: papillary thyroid carcinoma (PTC), tumor microenvironment, prognosis, N6-methylandenosine (m6A).

\section{Introduction}

In the past few decades, the incidence of thyroid cancer has sharply increased globally(H Lim, et al. 2017). Clinically, papillary thyroid carcinoma (PTC) is the most common histological subtype, accounting for up to 85\%(W Wang, et al. 2020). Most patients with PTC usually present with indolent tumors and have a favorable prognosis after receiving standardized treatment. Nevertheless, up to $20 \%-30 \%$ of patients with PTC experience recurrence or distant metastasis during follow-up(W Wang, et al. 2021, K Sugino, et al. 2020). Therefore, early detection and accurate management of the disease are vital for improving the prognosis. Unfortunately, the underlying molecular mechanisms that regulate PTC progression still unknown.

N6-methylandenosine (m6A) modification is the most prevalent post-transcriptional epigenetic modification of mRNA or non-coding RNAs (ncRNAs) in eukaryotic cells that modulating RNA stability, translation, splicing, and export (S Ma, et al. 2019, L He, et al. 2019, SD Kasowitz, et al. 2018). m6A modification is a reversible and dynamic process regulated by methyltransferases (m6A writers), binding proteins (m6A readers), and demethylases (m6A erasers) (Y Zhao, et al. 2020, Z Zhou, et al. 2020, T Wang, et al. 2020). The methyltransferase complex includes WTAP, METTL3, METTL14, RBM15, ZC3H13, KIAA1429, and RBM15B, which mediate the methylation modification process. Demethylases consist of $A L K B H 5$ and FTO. Readers are composed of $I G F 2 B P 1 / 2 / 3, Y T H D F 1 / 2 / 3, Y T H D C 1 / 2, H N R N P C$, and HNRNPA2B1. Increasing evidence has proved that $\mathrm{m} 6 \mathrm{~A}$ modification is closely associated with embryonic stem cell self-renewal, immune response, tissue development, and ncRNA processing( $Z$ Shulman, et al. 2020, YC Yi, et al. 2020, J Wen, et al. 2018). Recent studies have revealed that $\mathrm{m} 6 \mathrm{~A}$ modification also participate in the tumor occurrence and progression of various cancers, including hepatocellular, glioma, thyroid, colorectal, and breast cancers(Q Fang, et al. 2020, R Tian, et al. 2020, Z Tu, et al. 2020, N Xu, et al. 2020, RSP Huang, et al. 2020). For instance, the upregulated expression of METTL3 in hepatocellular carcinoma is positively related with poor prognosis, and METTL3mediated m6A modification led to epigenetic silencing of SOCS2 via an m6AYTHDF2-dependent mechanism(M Chen, et al. 2018). Moreover, IGF2BP3 overexpression has been observed in colorectal cancer and knockdown of IGF2BP3 represses angiogenesis and DNA replication through reading m6A modification of $V E G F$ and $C C N D 1$, respectively(Z Yang, et al. 2020). Additionally, the downregulated expression of METTL14 in four breast cancer subtypes can predict unfavorable prognosis(PJ Gong, et al. 2020). These studies suggest that m6A regulators are highly involved in malignant biological processes, thus serving as useful therapeutic targets with promising prognostic values. 
Several studies have highlighted that the aberrant expression of long non-coding RNAs (lncRNAs) also plays a critical role in cancer initiation and development of PTC, and dysregulation of lncRNA is closely related to the tumor development and progression (M Fan, et al. 2013, F Xia, et al. 2018). Another study has demonstrated that lncRNA GAS5 sponges miR-362-5p to upregulate $S M G 1$ to promote proliferation and invasion (L Li, et al. 2020). Upregulated lncRNA MALAT1 levels exacerbate cell growth and invasion by regulating microRNA (miR)-204(M Ye, et al. 2021). However, the underlying mechanism of m6A modification regulating the functions of lncRNA remains unclear, and no study has elucidated the role of m6A methylation-related lncRNAs (m6A-lncRNAs) in the biological functions involved in PTC progression and tumor microenvironment. Thus, a better understanding of the mechanisms of m6AlncRNAs involved in PTC tumorigenesis and progression may help determine effective biomarkers that can precisely predict prognosis and develop personalized immunotherapy for PTC management.

In the present study, we systematically explore the prognostic significance and tumor microenvironment heterogenicity of m6A-lncRNAs in PTC. This study may provide a new insight into the regulatory mechanisms that participate in tumor immune microenvironment and the treatment strategies for PTC.

\section{Materials and methods}

\section{Gene datasets and clinical data collection}

We downloaded the RNA-seq dataset containing 58 normal and 470 thyroid cancer samples from the TCGA database (https://portal.gdc.cancer.gov) with complete clinical information. The corresponding clinicopathological data including sex, age, multifocality, TNM stage, lymph node metastasis (LNM), histological type, extrathyroidal extension (ETE), bilaterality, and survival time were used for further analysis. According to previous publications, $20 \mathrm{~m} 6 \mathrm{~A}$ RNA methylation genes were identified, including writers (WTAP, METTL3, METTL14, METTL16, KIAA1429, ZC3H13, and RBM15), readers (HNRNPA2B1, IGF2BP1/2/3, YTHDC1/2, FMR1, LRPPRC, and YTHDF1/2/3), and erasers $(A L K B H 5$ and $F T O)$. Next, the differential expression of these genes was assessed in the PTC versus normal samples by using the "Limma" package. The workflow of the present study is illustrated in Figure 1.

\section{Risk assessment model construction}

First, univariate Cox regression analysis was performed to filter prognosis related lncRNAs $(P<0.05)$. Then, Pearson's correlation method was used to select the m6A-1ncRNA based on the threshold criteria of Pearson's coefficient $|\mathrm{R}|>0.6$ and $P<0.001$. A total of 19 prognostic m6A-1ncRNAs were extracted and analyzed. Then, an unsupervised clustering algorithm by using the $\mathrm{R}$ package "Consensus-ClusterPlus" was used to classify patients with PTC into different types of subgroups after conducting 1000 repetitions. Heatmaps were constructed by the Pearson distance measurement method and average linkage method. To further identify potential m6A-lncRNA regulators that affect prognosis, the least absolute shrinkage and selection operator (LASSO) Cox regression was performed to select candidate risk 
m6A-lncRNA regulators. The risk score for each patient was calculated according to the following algorithm: Risk score $==\sum_{i=1}^{n} \operatorname{coe} f(i) * a(i)$. The $\alpha i$ represented the expression level of m6A-lncRNAs, whereas coef $(i)$ represents the coefficient of each m6A-lncRNAs.Thereafter, the patients were divided into low-and high-risk groups based on the median value of the risk score. Kaplan-Meier curves with the log-rank test were used to draw the survival curves. The area under the ROC curve (AUC) was used to assess the predictive accuracy of the risk score model.

\section{Tumor immune estimation resource}

The immune and stroma scores for each patient were measured by the ESTIMATE algorithm via using the "estimate" $R$ package with default parameters, and tumor purity was calculated based on genomic methods. The association between clustering subtypes and risk score, and the abundance of six types of infiltrating immune cells, including CD8+ T cells, macrophages, CD4+ T cells, dendritic cells (DC), B cells, and neutrophils, was calculated using the Tumor Immune Estimation Resource (TIMER) algorithm.

\section{Enrichment functional analysis}

Differentially expressed genes (DEGs) between the high- and low-risk subgroups were screened using the "limma" package based on $P$ values $<0.05$ and $|\log 2 \mathrm{FC}| \geqslant 1$. Kyoto Encyclopedia of Genes and Genomes (KEGG) and gene ontology (GO) analysis was performed by using the "clusterProfiler" $\mathrm{R}$ package for pathway and functional enrichment analysis. On the other hand, gene set enrichment analysis (GSEA) was performed to identify significant pathways in the high-risk subgroup compared with the low-risk subgroup.

\section{CeRNA network construction}

First, the $7 \mathrm{~m} 6 \mathrm{~A}-1 \mathrm{ncRNAs}$ interacting with miRNAs were predicted using the miRcode database. Then, we predicted target mRNAs for miRNAs by using miRTarBase (http://mirtarbase.mbc.nctu.edu.tw/php/index.php), miRDB (http://mirdb.org), and TargetScan (http://www.targetscan.org). Finally, a lncRNA-miRNA-mRNA ceRNA network was created and visualized using the alluvial plot (http://www.bioinformatics.com.cn).

\subsection{Quantitative real-time PCR validation}

We collected 30 PTC tissue samples and paired adjacent normal tissue samples from patients who underwent thyroidectomy in the Thyroid Surgery Department of Xiangya hospital from March 2020 to July 2020. The fresh tissues were stored at $-80^{\circ} \mathrm{C}$. Informed consent was obtained from all the participants and this study was approved by the Ethics Committee of Xiangya Hospital of Central South University. Besides, four human thyroid cancer cell lines (BCPAP, K1, TPC-1, IHH4) and human normal thyroid epithelial cell line (nthy-ori3-1) were used and cultured in a complete medium composed of $10 \%$ fetal bovine serum (Gibco) and 90\% RPMI1640 (Gibco, Carlsbad, USA) or DMEM (Gibco, Carlsbad, USA), supplemented with $100 \mathrm{U} / \mathrm{mL}$ penicillin (HyClone) and $100 \mathrm{mg} / \mathrm{mL}$ streptomycin. These cells were cultured in $37{ }^{\circ} \mathrm{C}$ with $95 \% \mathrm{O}_{2}$ and $5 \% \mathrm{CO}_{2}$.

Briefly, we used reverse transcription to construct the first strand of cDNA using 100 
ng of total RNA according to the manufacturer's instructions, Then, quantitative realtime PCR (qRT-PCR) analysis was performed using TBGreen Premix Ex TaqTMII (Cat \# RR047A-5, TaKaRa, Japan). Primer sequences for m6A-lncRNAs are listed in Supplementary Table 1. Experiments were performed in triplicate.

\section{Statistical analysis}

Statistical tests were performed using SPSS 22.0 (IBM, NY, USA) and R version 3.6.0. Chi-square and Student's $t$-tests were performed to compare the differences between the two subgroups. The Kruskal-Wallis test was used to compare immune scores, stroma scores, tumor purity, and ESTIMATE scores among different cluster subgroups. Survival curves were depicted by using the Kaplan-Meier method. In addition, Univariate and multivariate Cox regression analyses to identify independent prognostic factors and establish an integrated nomogram combining predictable clinicopathological factors and risk scores. The predictive performance of the nomogram was validated by calibrated plots and the concordance index (C index). The receiver operating characteristic (ROC) curve was used to verify the prognostic ability of the nomogram for 3-/5-year OS and decision curve analysis was employed to assess the clinical values. The statistical significance was shown as followed: $P$ $<0.05(*), P<0.01(* *), P<0.001(* * *)$.

\section{Results}

\section{The profile of m6A -IncRNA regulators}

To determine the biological role of m6A-lncRNAs in the development of PTC, we first systematically explored the expression profiles of 20 m6A regulatory genes in PTC and the corresponding normal samples in the TCGA datasets. The expression of $A L K B H 5$, FTO, METTL3, METTL14, WTAP, YTHDF1, YTHDF3, YTHDC2, YTHDC1, ZC3H13, HNRNPA2B1, RBM15, IGF2BP1, IGF2BP3, and LRPPRC was significantly downregulated in PTC than in the normal samples $(P<0.05)$, whereas that of IGF2BP2 $(P<0.001)$ and HNRNPC $(P<0.001)$ was remarkably upregulated in PTC (Figure 2A). On the other hand, Pearson correlation analysis showed that 185 lncRNAs were significantly associated with m6A RNA methylation genes. Subsequently, univariate cox regression analysis was performed to screen prognosis-related m6A-lncRNAs and found that $19 \mathrm{~m} 6 \mathrm{~A}-\mathrm{lncRNAs}$ were significantly associated with the OS. Fig 2B showed that most of m6A regulators positively correlated with the expression level of lncRNAs. These results indicated that $\mathrm{m} 6 \mathrm{~A}-\mathrm{lncRNA}$ regulators are reliable factors for predicting prognosis.

\section{Consensus clustering of m6A-IncRNA regulators with prognosis and the tumor immune microenvironment}

Next, according to the expression similarity of m6A-lncRNAs, $\mathrm{k}=3$ was considered as the optimal selection with the clustering stability increasing from $\mathrm{k}=2$ to 9 (Figure S1). Therefore, a total of 470 PTC patients with complete clinical parameters were classified into three subgroups: cluster $1(\mathrm{n}=141)$, cluster $2(\mathrm{n}=158)$, and cluster $3(\mathrm{n}=171)$. As shown in the heatmap of cluster analysis, 19 m6A-lncRNAs could be identified in different samples. We also found significant differences in the TNM stage, histological subtype, T stage, ETE, and LNM (all $P<0.001$ ) among the three clusters (Figure $3 \mathrm{~A})$. Moreover, the OS of cluster 1 was significantly shorter than that of the other two 
clusters ( $p=0.033$, Figure 3B).

To better understand the effect of m6A-lncRNAs on the tumor immune microenvironment, we further evaluated the infiltration level of immune cells and immune scores among the three clusters. As shown in Figure 3C, ESTIMATE, immune score, and stroma score were markedly decreased, whereas the tumor purity score was significantly increased in cluster 1 than in the other two clusters $(P<0.001)$, indicating that cluster 1 was characterized by reduced immune activity. Additionally, the abundance of B cells, CD8+ T cells, and macrophages were relatively higher, as well as the relatively lower enrichment of CD4+ T cells, neutrophils, and DC in cluster 1 (Figure 3D).

Risk score was associated with PTC prognosis and tumor immune microenvironment

To establish risk scores to predict the OS of patients with PTC, the LASSO Cox regression analysis was performed to further to screen out prognosis-related m6AlncRNAs, 8 m6A-lncRNAs exhibited strong prognostic value (Figure S2). The risk score for each patient was calculated according to the following algorithm:

Risk score $=(0.536 \times A C 139795.2)+(0.131 \times$ TRAM2.AS1 $)+(0.559 \times P O L R 2 J 4)+$

$\times N O R A D)+(0.676 \times A L 022328.2)$, the patients were divided into low- and

high-risk subgroups based on the median risk score. Principal component analysis could distinct the distribution of the two risk groups (Figure S3).and the distributions of the risk score, OS, and OS status of each PTC patient are shown in Figure 4A, Heatmap distribution showed that PTC patients with LNM, ETE, classical histological subtype, and T3-4 stage had higher risk scores $(P<0.05$, Figure 4B). Survival analysis indicated patients in the high-risk subgroup had worse OS compared with those in the low-risk subgroup (Figure 4C), and the risk score model exhibited a good prediction performance with the area under the curve (AUC) of 0.8021 (Figure 4D), suggesting that the risk score model based on 8 m6A-lncRNAs accurately predicted the prognosis.

We also determined the relationship between the risk score and the tumor immune microenvironment. The ESTIMATE and immune scores were notably higher ( $P$ $<0.001$ ), whereas the tumor purity score was significantly lower in the high-risk subgroup than in the low-risk subgroup. In cluster 1, which had a worse prognosis, the risk score was significantly higher than the other two clusters (Figure 4E, $p<0.001$ ). Moreover, the abundance of neutrophils, DCs, and CD4+ T cells were higher but that of CD8+ T cells and macrophages was distinctly lower in the low-risk subgroup than in the high-risk subgroup (Figure 4F). These data indicated that the tumor immune microenvironment plays a critical role in PTC tumorigenesis.

GSEA, and pathway and functional enrichment analysis

To better comprehend the potential biological mechanisms between the high- and low- 
risk score subgroups, we identified DEGs based on the criterion $P<0.05$ and $\log _{2} \mathrm{FC}>$ 1 and subsequently implement the KEGG pathway and GO function analysis. The top 5 GO terms used were NABA matrisome associated, thyroid hormone synthesis, surfactant metabolism, regulated exocytosis, and interleukin-4 and interleukin-13 signaling. (Figure 5A).

Furthermore, GSEA revealed that the malignant hallmarks of cancer, including Wnt $/ \beta$ catenin signaling, HEME metabolism, UV response, KRAS signaling, bile acid metabolism, and MITOTIC spindle were closely associated with the high-risk subgroups (Figure 5B). These results cumulatively prove that the risk score was significantly associated with the biological mechanisms of PTC.

\section{Construction of a prognostic nomogram for PTC}

We also implemented univariate and multivariate analyses to investigate the independent prognostic factors for PTCs. The forest plots revealed that the TNM stage $(P<0.01)$, age $(P<0.001)$, and risk sore $(P<0.001)$ were significantly correlated with OS in the univariate analysis, while the results of multivariate Cox regression analysis indicated age $(\mathrm{OR}=1.15 ; 95 \% \mathrm{CI}, 1.08-1.22$; and $P<0.001)$ and risk sore $(\mathrm{OR}=$ $2.16 ; 95 \% \mathrm{CI}, 1.37-3.42$; and $P<0.001)$ to act as independent prognostic factors (Table 1).

Furthermore, to meet the requirement for clinicians to easily evaluate the prognosis of PTC patients, we formulated an integrated nomogram based on the independent prognostic factors for calculating the individual OS (Figure 6A). The Cindex was 0.923, compared with TNM stage, this nomogram model has better predictive performance (AUC: 0.743 vs.0.963) (Figure 6C). The calibration plots demonstrated good accuracy in predicting the 3- and 5-year OS (Figure 6B). Decision curve analysis (DCA) demonstrated that the integrated nomogram had an excellent net benefit compared with the risk score model and age (Figure 6D). These data suggested that the nomogram can better predict the OS of patients with PTC.

\section{The ceRNA network for PTC}

First, 39 miRNAs were extracted from the miRcode database based on 7 m6A-lncRNAs. Next, three predictive databases (miRDB, TargetScan, and miRTarBase) were used to search target mRNAs and 72 mRNAs were identified. Finally, 7 m6A-lncRNAs, 39 miRNAs, and 72 mRNAs were included in the ceRNA network (Figure 7A). Moreover, 72 target mRNAs were used to perform functional and pathway enrichment analysis and the results indicated that these target genes were enriched in the cellular response to glucocorticoid stimulus, skeletal system development, embryonic eye morphogenesis, negative regulation of cell differentiation, rhythmic process, insulin signaling pathway, molecules associated with elastic fibers, and transcriptional misregulation in cancer (Figure 7B). These results may provide some potential insight into understanding the role of these m6A-lncRNAs in PTC tumorigenesis.

\subsection{Validation of m6A-IncRNAs expression in PTC tissue samples}

To validate the results of bioinformatics analysis, RT-qPCR was performed on PTC samples and cell lines. The expression level of AC018653-3, GABPB1-AS1, and NORAD were downregulated in PTC samples compared to normal thyroid tissue samples, and only DOCK9-DT was significantly upregulated in PTC samples. 
TRAM2-AS1, POLR2J4, and AC139795.2 showed no significant differences (Figure 8A). Besides, the expression level of NORAD and GABPB 1-AS1 were significantly up-regulated in PTC cell lines compared with normal thyroid epithelial cell (all $p<$ 0.05). While $A C 018653$-3 and $A C 139795.2$ were significantly down-regulated in PTC cell lines (both $p<0.05$ ). However, the expression of TRAM2-AS1, DOCK9-DT, and POLR2J4 showed no significant differences (Figure 8B).

\section{Discussion}

The molecular pathogenesis and development of PTC have been attributed to various factors, including the abnormal expression of tumor suppressor genes and oncogenes, exposure to external radiation, and genetic mutations. m6A RNA methylation is a nascent field of research but is garnering considerable scientific attention. Increasing evidence has shown that m6A RNA methylation can target or modulate lncRNA to affect cancer initiation and development (YC Yi, XY Chen, J Zhang JS Zhu 2020, Z Tu, et al. 2020, S Wen, et al. 2020). To the best of our knowledge, this is the first study to systematically elucidate the potential contribution of m6A-lncRNA regulators in the prognosis of PTC and specifically highlight their role in the tumor immune microenvironment. Our findings have provided a novel insight into the regulatory mechanisms that govern the tumor immune microenvironment based on which the treatment strategies for PTC can be developed.

Several studies have demonstrated that m6A modification plays a pivotal role in the pathological processes of carcinoma development (J Wang, et al. 2020, L Zhang, et al. 2020, K Guan, et al. 2020); however, its role in the lncRNA-dependent development of PTC remains unclear. In glioblastoma, $A L K B H 5$ interacts with the lncRNA FOXM1- $A S$,

which enhances the demethylation of the 3' UTRs of FOXM1 transcripts to promote tumor proliferation and tumorigenesis(S Zhang, et al. 2017). In pancreatic cancer, $I G F 2 B P 2$ has been implicated as a reader to regulate lncRNA DANCR, leading to cell viability and proliferation, and stemness-like properties (X Hu, et al. 2020). IGF2BP2 also directly binds to $P D X 1$ in an m6A-dependent manner and promotes pancreatic $\beta$ cell proliferation in type 2 diabetes (L Regue, et al. 2021). Moreover, lncRNA might act as ceRNA, targeting m6A-related genes and thereby affecting the progression of aggressive tumors ( $Z$ Yang, et al. 2020). In this study, we performed Pearson's correlation analysis to mine m6A-related lncRNA and identified three subgroups by consensus clustering: cluster 1 , cluster 2 , and cluster 3 , respectively. These clusters not only affected the prognosis of PTC patients but were also closely associated with the TNM stage, histological subtype, T stage, ETE, and LNM (all $P<0.001$ ). Moreover, we established a risk score model based on the expression of 8 m6A-lncRNAs, which effectively stratified PTC patients into high- and low-risk groups. Patients with a high-risk score exhibited poor prognoses, PTC patients with LNM, ETE, and T3-4 stage simultaneously presented higher risk scores. The risk stratification based on risk score could facilitate the determination of therapeutic options to improve prognoses.

Besides, the tumor immune microenvironment has received extensive attention. The $\mathrm{TME}$ is formed in a process of dynamic changes and regulated by immune editing(X 
Wang, et al. 2021, I Kaymak, et al. 2021). The unbalance of TME can lead to the occurrence and development of disease(Z Xie, et al. 2020). However, the underlying mechanism of m6A modification on TME in PTC remains unclear. In this study, the risk score based on the eight m6A-lncRNAs were significantly correlated with immune cells infiltration. Compared with low-risk group, immune, stroma, and ESTIMATE scores were significantly downregulated in high-risk group. Moreover, the abundance of immune cells such as neutrophils, DCs, and CD4+ T cells was highly infiltrated in the low-risk group. As mentioned before, survival analysis confirmed that patients in cluster 1 had an unfavorable prognosis. Corresponding immune infiltration scores were decreased markedly, whereas the tumor purity score was significantly increased in cluster 1 than in the other two clusters $(P<0.001)$. These findings indicated that the tumor immune microenvironment deeply participated in the tumorigenesis of PTC. Currently, the prognostic prediction and risk stratification for PTC patients is mainly dependent on the TNM scoring system, which is cumbersome and cannot accurately predict patient prognosis(SA Ghaznavi, et al. 2018, BR Haugen, et al. 2016). Considering the heterogeneity in PTC, several strong prognostic biomarkers such as $B R A F^{V 600 E}$, TERT promoter, and RAS mutation have been widely reported(L Zhao, et al. 2020, J Park, et al. 2021). However, these potential biomarkers are not sufficiently sensitive and lack the accuracy in predicting the long-term survival rate in clinical practice. To improve the accuracy of the survival prognostic model, we established an integrated nomogram by combining the predictable clinicopathological factors with the m6A-lncRNA risk scores. The calibration plots showed good accuracy in predicting the 3- and 5-year OS. Compared with TNM stage, our survival prediction model has better predictive performance (AUC: 0.743 vs.0.963). In addition, we constructed a ceRNA network based on the 7 m6A-lncRNAs, 39 miRNAs, and $72 \mathrm{mRNAs}$, which provided some potential insight into understanding the role of these m6A-lncRNAs in PTC tumorigenesis.

Finally, we validated the mRNA expression of the prognostic m6A-lncRNAs in PTC samples and cell lines for subsequent functional and molecular experiments. Considering the expression level of m6A-lncRNAs, NORAD and GABPB1-AS1 were the most meaningful signatures for further research. Previous studies demonstrated that NORAD promoted tumor proliferation and progression in nonsmall-cell lung cancer(Q Huang, et al. 2020), endometrial cancer(T Han, et al. 2020), and melanoma(Y Chen, et al. 2019). By contrast, NORAD serve as suppressor genes in neuroblastoma(Y Yu, et al. 2020) and breast cancer(W Liu, et al. 2021), respectively, which was consistent with our results. Li et al(X Li, et al. 2021) reported GABPB1-AS1 competitively bound to miR-330 and reinforced ZNF367 expression, leading to facilitate glioma cells progression. In cervical cancer, E6induced GABPB1-AS1 overexpression facilitated tumor proliferation and invasion(R $\mathrm{Ou}$, et al. 2020). However, the function and mechanism of NORAD and GABPB1-AS1 in thyroid cancer have not been reported, and its role in PTC needed further exploration. Undeniably, there are several limitations in the present study. First, our findings are based on TCGA databases without our cohort. resulting in an inevitable selection bias in clinical and genetic data. Second, because of limited project funding, we only used 
RT-qPCR to validate the level expression of m6A-lncRNAs, including cellular function- and regulation mechanism-based studies, are still needed. Third, the prognostic predictive model was based on the TCGA cohort with a small sample size, and the interactions between the TME and m6A-lncRNAs are also not experimentally validated because of the lack of sufficient available datasets. Fouth, the correlation between m6A regulators and lncRNA have been analyzed, lacking of experiments such as MeRIP-seq, m6A-IP-qPCR, and RNA-seq to further confirm m6A modification sites on IncRNA. Last, but not least, important clinical information, such as the treatment strategy (radioactive iodine ablation), TERT promoter and $B R A F^{V 600 E}$ mutation, and esophagus and tracheal invasions, was not available. Hence, future clinical and experimental studies are necessary to validate the application of our survival prediction model in clinical practice.

\section{Conclusion}

In summary, this study systematically assessed prognostic value, role in the TME, and potential regulatory mechanisms of m6A-lncRNAs in PTC. Three PTC subtypes were determined via the consensus clustering and the risk score developed from $8 \mathrm{~m} 6 \mathrm{~A}$ lncRNAs that stratified the prognosis and presented the significantly different TME. This is the first study to reveal that m6A-lncRNAs play a vital role in the prognosis and TME of PTC. To a certain degree, m6A-lncRNAs can be considered as new, promising prognostic biomarkers and treatment targets. Our findings also provided crucial insight to support further research regarding the role of m6A-lncRNAs in PTC development.

\section{Acknowledgments}

We thank the TCGA database for gene expression and clinical data.

\section{Author Contributions}

XL, WW, and QS conceived the study, designed the research, and wrote the paper. XL, WW, BT, and YZ conducted and analyzed experiments. XQ, and SY provided samples. XL supervised the research. All authors contributed to the article and approved the submitted version.

\section{Funding}

This work was supported by the National Natural Science Foundation of China (grant No. 81672885), the Natural Science Foundation of Hunan Province (grant No. 2019JJ40500) and Innovative Foundation for graduate students of Hunan Province (grant No. 2020zzts259).

\section{Availability of data and materials}

All data not included in the manuscript is available from the supplementary materials.

\section{Ethics approval and consent to participate}

Informed consent was obtained from all the participants and this study was approved by the Ethics Committee of Xiangya Hospital of Central South University.

\section{Consent for publication}

All authors read and agreed to the content of the final manuscript, and consented to publish this material. 


\section{Competing interests}

The authors declare that they have no competing interests.

\section{References}

1. Lim H., Devesa SS., Sosa JA., Check D., Kitahara CM. Trends in Thyroid Cancer Incidence and Mortality in the United States, 1974-2013. JAMA.2017; 317:1338-1348.

2. Wang W., Yang Z., Ouyang Q. A nomogram to predict skip metastasis in papillary thyroid cancer. World J Surg Oncol.2020; 18:167.

3. Wang $W$, et al. Identification and validation of potential novel biomarkers to predict distant metastasis in differentiated thyroid cancer. Annals of Translational Medicine.2021; 9:1053-1053.

4. Sugino K, et al. Distant Metastasis in Pediatric and Adolescent Differentiated Thyroid Cancer: Clinical Outcomes and Risk Factor Analyses. J Clin Endocrinol Metab.2020; 105:

5. Ma S, et al. The interplay between m6A RNA methylation and noncoding RNA in cancer. J Hematol Oncol.2019; 12:121.

6. He L, et al. Functions of N6-methyladenosine and its role in cancer. Mol Cancer.2019; 18:176.

7. Kasowitz SD, et al. Nuclear m6A reader YTHDC1 regulates alternative polyadenylation and splicing during mouse oocyte development. PLoS Genet.2018; 14:e1007412.

8. Zhao Y., Shi Y., Shen H., Xie W. m(6)A-binding proteins: the emerging crucial performers in epigenetics. J Hematol Oncol.2020; 13:35.

9. Zhou Z, et al. Mechanism of RNA modification N6-methyladenosine in human cancer. Mol Cancer.2020; 19:104.

10. Wang T., Kong S., Tao M., Ju S. The potential role of RNA N6 - methyladenosine in Cancer progression. Mol Cancer.2020; 19:88.

11. Shulman Z., Stern-Ginossar N. The RNA modification N(6)-methyladenosine as a novel regulator of the immune system. Nat Immunol.2020; 21:501-512.

12. Yi YC., Chen XY., Zhang J., Zhu JS. Novel insights into the interplay between m(6)A modification and noncoding RNAs in cancer. Mol Cancer.2020; 19:121.

13. Wen J, et al. Zc3h13 Regulates Nuclear RNA m(6)A Methylation and Mouse Embryonic Stem Cell Self-Renewal. Mol Cell.2018; 69:1028-1038 e1026.

14. Fang Q., Chen $\mathrm{H}$. The significance of m6A RNA methylation regulators in predicting the prognosis and clinical course of HBV-related hepatocellular carcinoma. Mol Med.2020; 26:60.

15. Tian R, et al. M6A Demethylase FTO Plays a Tumor Suppressor Role in Thyroid Cancer. DNA Cell Biol.2020;

16. Tu Z, et al. N6-Methylandenosine-Related IncRNAs Are Potential Biomarkers for Predicting the Overall Survival of Lower-Grade Glioma Patients. Front Cell Dev Biol.2020; 8:642.

17. Xu N., Chen J., He G., Gao L., Zhang D. Prognostic values of m6A RNA methylation regulators in differentiated Thyroid Carcinoma. J Cancer.2020; 11:5187-5197.

18. Huang RSP, et al. Biomarkers in Breast Cancer: An Integrated Analysis of Comprehensive Genomic Profiling and PD-L1 Immunohistochemistry Biomarkers in 312 Patients with Breast Cancer. Oncologist.2020; 25:943-953.

19. Chen M, et al. RNA N6-methyladenosine methyltransferase-like 3 promotes liver cancer 
progression through YTHDF2-dependent posttranscriptional silencing of SOCS2. Hepatology.2018; 67:2254-2270.

20. Yang Z, et al. RNA N6-methyladenosine reader IGF2BP3 regulates cell cycle and

21. Gong PJ, et al. Analysis of N6-Methyladenosine Methyltransferase Reveals METTL14 and

22. Fan $\mathrm{M}$, et al. A long non-coding RNA, PTCSC3, as a tumor suppressor and a target of miRNAs in thyroid cancer cells. Exp Ther Med.2013; 5:1143-1146.

23. Xia $F$, et al. Long Noncoding RNA HOXA-AS2 Promotes Papillary Thyroid Cancer Progression by Regulating miR-520c-3p/S100A4 Pathway. Cell Physiol Biochem.2018; 50:1659-1672.

24. Li L., Lin X., Xu P., Jiao Y., Fu P. LncRNA GAS5 sponges miR-362-5p to promote sensitivity of thyroid cancer cells to (131) I by upregulating SMG1. IUBMB Life.2020; 72:2420-2431.

25. Ye M., Dong S., Hou H., Zhang T., Shen M. Oncogenic Role of Long Noncoding RNAMALAT1 in Thyroid Cancer Progression through Regulation of the miR204/IGF2BP2/m6A-MYC Signaling. Mol Ther Nucleic Acids.2021; 23:1-12.

26. Wen S, et al. Long non-coding RNA NEAT1 promotes bone metastasis of prostate cancer through N6-methyladenosine. Mol Cancer.2020; 19:171.

27. Wang J., Zhang C., He W., Gou X. Effect of m(6)A RNA Methylation Regulators on Malignant Progression and Prognosis in Renal Clear Cell Carcinoma. Front Oncol.2020; 10:3.

28. Zhang L, et al. Expression Pattern and Prognostic Value of Key Regulators for m6A RNA

29. Guan K, et al. Expression Status And Prognostic Value Of M6A-associated Genes in Gastric Cancer. J Cancer.2020; 11:3027-3040.

30. Zhang S, et al. m(6)A Demethylase ALKBH5 Maintains Tumorigenicity of Glioblastoma Stem-like Cells by Sustaining FOXM1 Expression and Cell Proliferation Program. Cancer Cell.2017; 31:591-606 e596.

31. Hu X, et al. IGF2BP2 regulates DANCR by serving as an N6-methyladenosine reader. Cell Death Differ.2020; 27:1782-1794.

32. Regue L, et al. RNA m6A reader IMP2/IGF2BP2 promotes pancreatic beta-cell proliferation and insulin secretion by enhancing PDX1 expression. Mol Metab.2021; 101209.

33. Yang Z, et al. ZFAS1 Exerts an Oncogenic Role via Suppressing miR-647 in an m(6)ADependent Manner in Cervical Cancer. Onco Targets Ther.2020; 13:11795-11806.

34. Wang $X$, et al. Identification of an immune-related signature indicating the dedifferentiation of thyroid cells. Cancer Cell Int.2021; 21:231.

35. Kaymak I., Williams KS., Cantor JR., Jones RG. Immunometabolic Interplay in the Tumor Microenvironment. Cancer Cell.2021; 39:28-37.

36. Xie Z, et al. Immune Cell Confrontation in the Papillary Thyroid Carcinoma Microenvironment. Front Endocrinol (Lausanne).2020; 11:570604.

37. Ghaznavi SA, et al. Using the American Thyroid Association Risk-Stratification System to Refine and Individualize the American Joint Committee on Cancer Eighth Edition DiseaseSpecific Survival Estimates in Differentiated Thyroid Cancer. Thyroid.2018; 28:1293-1300. 8. Haugen BR, et al. 2015 American Thyroid Association Management Guidelines for Adult 
Patients with Thyroid Nodules and Differentiated Thyroid Cancer: The American Thyroid Association Guidelines Task Force on Thyroid Nodules and Differentiated Thyroid Cancer. Thyroid.2016; 26:1-133.

39. Zhao L, et al. The Coexistence of Genetic Mutations in Thyroid Carcinoma Predicts Histopathological Factors Associated With a Poor Prognosis: A Systematic Review and Network Meta-Analysis. Front Oncol.2020; 10:540238.

40. Park J, et al. TERT Promoter Mutations and the 8th Edition TNM Classification in Predicting the Survival of Thyroid Cancer Patients. Cancers.2021; 13:648.

41. Huang Q., Xing S., Peng A., Yu Z. NORAD accelerates chemo-resistance of non-small-cell lung cancer via targeting at miR-129-1-3p/SOX4 axis. Biosci Rep.2020; 40:

42. Han T, et al. NORAD orchestrates endometrial cancer progression by sequestering FUBP1 nuclear localization to promote cell apoptosis. Cell Death Dis.2020; 11:473.

43. Chen $Y$, et al. Overexpression of long non-coding RNA NORAD promotes invasion and migration in malignant melanoma via regulating the MIR-205-EGLN2 pathway. Cancer Med.2019; 8:1744-1754.

44. Yu Y, et al. Downregulated NORAD in neuroblastoma promotes cell proliferation via chromosomal instability and predicts poor prognosis. Acta Biochim Pol.2020; 67:595-603.

45. Liu W., Zhou X., Li Y., Jiang H., Chen A. Long Non-Coding RNA NORAD Inhibits Breast Cancer Cell Proliferation and Metastasis by Regulating miR-155-5p/SOCS1 Axis. J Breast Cancer.2021; 24:330-343.

46. Li X., Wang H. Long Non-Coding RNA GABPB1-AS1 Augments Malignancy of Glioma Cells by Sequestering MicroRNA-330 and Reinforcing the ZNF367/Cell Cycle Signaling Pathway. Neuropsychiatr Dis Treat.2021; 17:2073-2087.

47. Ou R, et al. HPV16 E6 oncoprotein-induced upregulation of IncRNA GABPB1-AS1 facilitates cervical cancer progression by regulating miR-519e-5p/Notch2 axis. FASEB J.2020; 34:13211-13223.

\section{Figure legends}

Figure 1: Study flow chart.

Figure 2: The profile of m6A-lncRNA regulators. A: Differential expression of $20 \mathrm{~m} 6 \mathrm{~A}$ RNA regulators; B: the correlations between m6A-related lncRNAs and m6A-related genes. ${ }^{*} p<0.05, * * p<0.01$, and $* * * p<0.001$.

Figure 3: Prognosis and tumor immune microenvironment in consensus clustering. A: heatmap of cluster analysis clinicopathologic features; B: the OS of three subgroups; C-D: immunoscore and immune cell infiltration levels of cluster1/2/3 subtypes. OS: overall survival; LNM; lymph node metastasis; ETE: extrathyroidal extension. ${ }^{*} p<0.05,{ }^{*} p p<0.01,{ }^{* * *} p<0.001$, and ns: no significance.

Figure 4: Construction and analysis of prognostic signatures of m6A -lncRNA regulators. A: the distributions of risk score, OS and OS status; B: the heatmap analysis the clinicopathologic features and eight m6A-lncRNA between low- and high-risk groups; C: Kaplan-Meier curves of OS by risk score group; D: the AUC value of the risk score; E-F: the immune cell infiltration landscape in the risk score subgroups. OS: overall survival; AUC: the area of ROC curve. LNM; lymph node metastasis; ETE: extrathyroidal extension. ${ }^{*} p<0.05,{ }^{*} p<0.01,{ }^{* * *} p<$ 
0.001, and ns: no significance.

558 Figure 5: GSEA and Pathway and Functional enrichment analysis. A: KEGG pathway and GO function analysis; B: GSEA showed results in the high-risk group. KEGG: Kyoto Encyclopedia of Genes and Genomes; GO: Gene ontology; GSEA: Gene set enrichment analysis.

Figure 6: Construction and evaluation of prognostic nomogram model. A: Nomogram based on age and risk score for 3- and 5-year OS prediction; B: the calibration plots of nomogram model; $\mathrm{C}$ : the AUC value of the nomogram and AJCC TNM stage.D: decision curve analysis evaluated OS benefits. OS: overall survival.

Figure 7: The ceRNA network for PTC. 7A: ceRNA network was constracted by seven m6A-lncRNA, thirty-nine miRNAs and seventy-two mRNAs. 7B: functional and pathways enrichment analysis for seventy-two mRNAs. Figure 8: Validation the expression level of m5C-lncRNAs. A-B: the expression level of m5C-lncRNAs in 30 pairs PTC tissues and nine cells line. ${ }^{*} p<0.05,{ }^{*} p<0.01$, $* * * p<0.001$, and ns: no significance.

Table 1 Univariate and multivariate Cox regression analysis the prognosis factors of PTC

\begin{tabular}{|c|c|c|c|c|}
\hline \multirow[t]{2}{*}{ Variable } & \multicolumn{2}{|c|}{ Univariate analysis } & \multicolumn{2}{|c|}{ Multivariate analysis } \\
\hline & HR (95\%CI) & $P$ & OR $(95 \% \mathrm{CI})$ & $P$ \\
\hline Age & $1.17(1.10-1.23)$ & $<0.001$ & $1.15(1.08-1.22)$ & $<0.001$ \\
\hline \multicolumn{5}{|l|}{ Gender } \\
\hline Male & ref & & - & \\
\hline Female & $0.47(0.17-1.32)$ & 0.15 & - & - \\
\hline \multicolumn{5}{|l|}{ TNM stage } \\
\hline I & ref & & ref & \\
\hline II & $5.67(0.80-40.20)$ & 0.083 & $1.74(0.23-12.99)$ & 0.589 \\
\hline III & $10.27(2.13-49.50)$ & 0.004 & $0.74(0.13-4.18)$ & 0.729 \\
\hline IV & $14.19(2.59-77.80)$ & 0.002 & $1.67(0.29-9.73)$ & 0.567 \\
\hline \multicolumn{5}{|l|}{ Multifocality } \\
\hline Unifocal & ref & & - & - \\
\hline Multifocal & $0.28(0.06-1.23)$ & 0.092 & - & - \\
\hline \multicolumn{5}{|l|}{ Bilaterality } \\
\hline Unilateral & ref & & - & - \\
\hline Bilateral & $1.20(0.26-5.44)$ & 0.810 & - & - \\
\hline Isthmus & $1.16(0.15-8.95)$ & 0.890 & - & - \\
\hline Risk score & $4.23(2.53-7.08)$ & $<0.001$ & $2.16(1.37-3.42)$ & $<0.001$ \\
\hline \multicolumn{5}{|c|}{ Pathological type } \\
\hline Other & ref & & - & - \\
\hline PTC & $4.74(0.62-36.10)$ & 0.130 & - & - \\
\hline
\end{tabular}


Figures

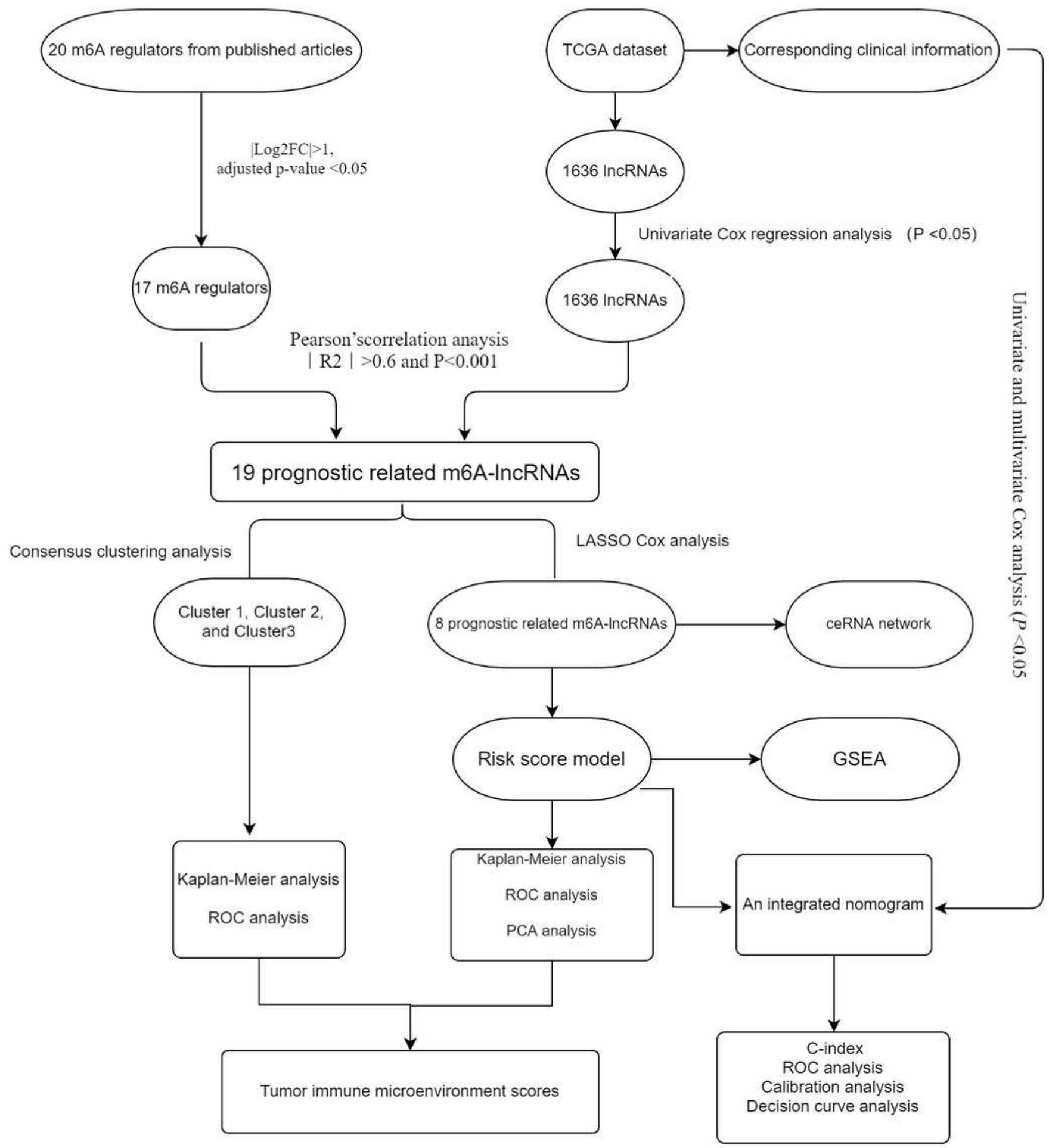

\section{Figure 1}

Study flow chart. 
A

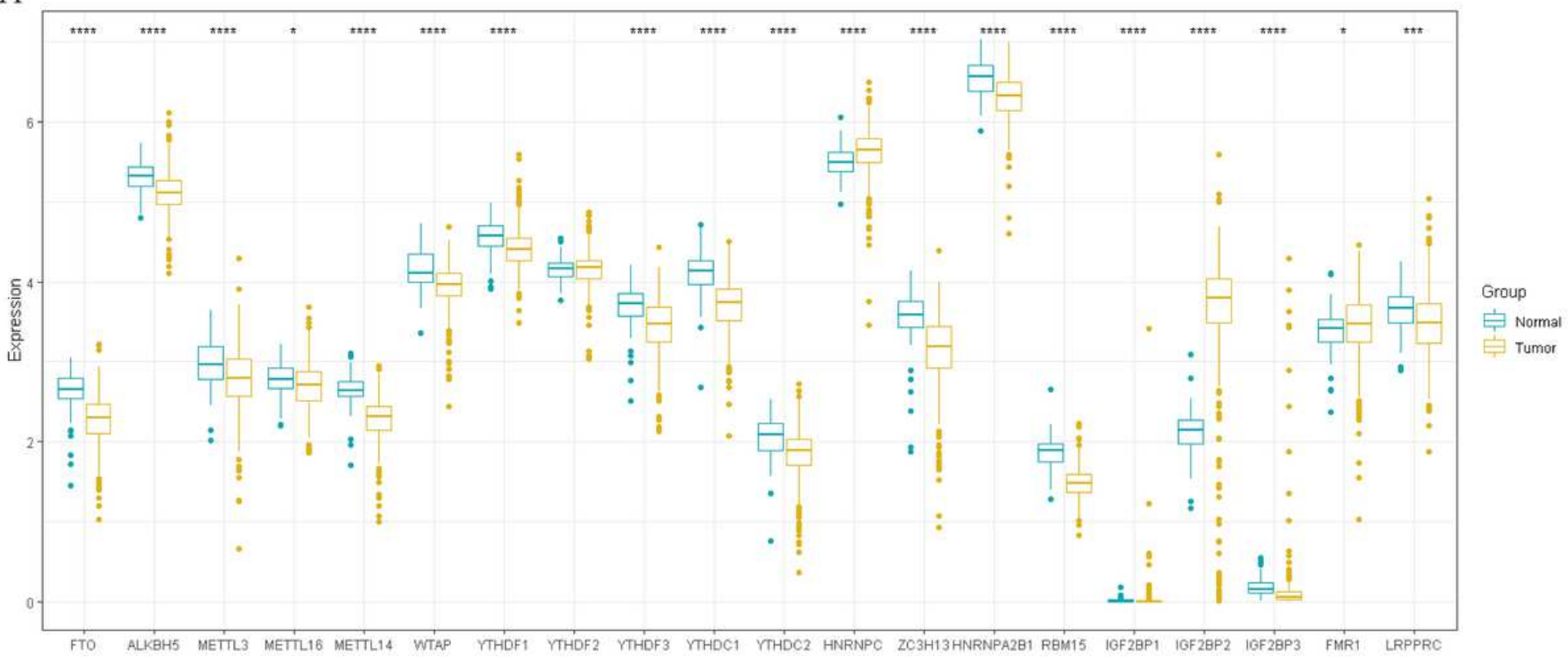

B

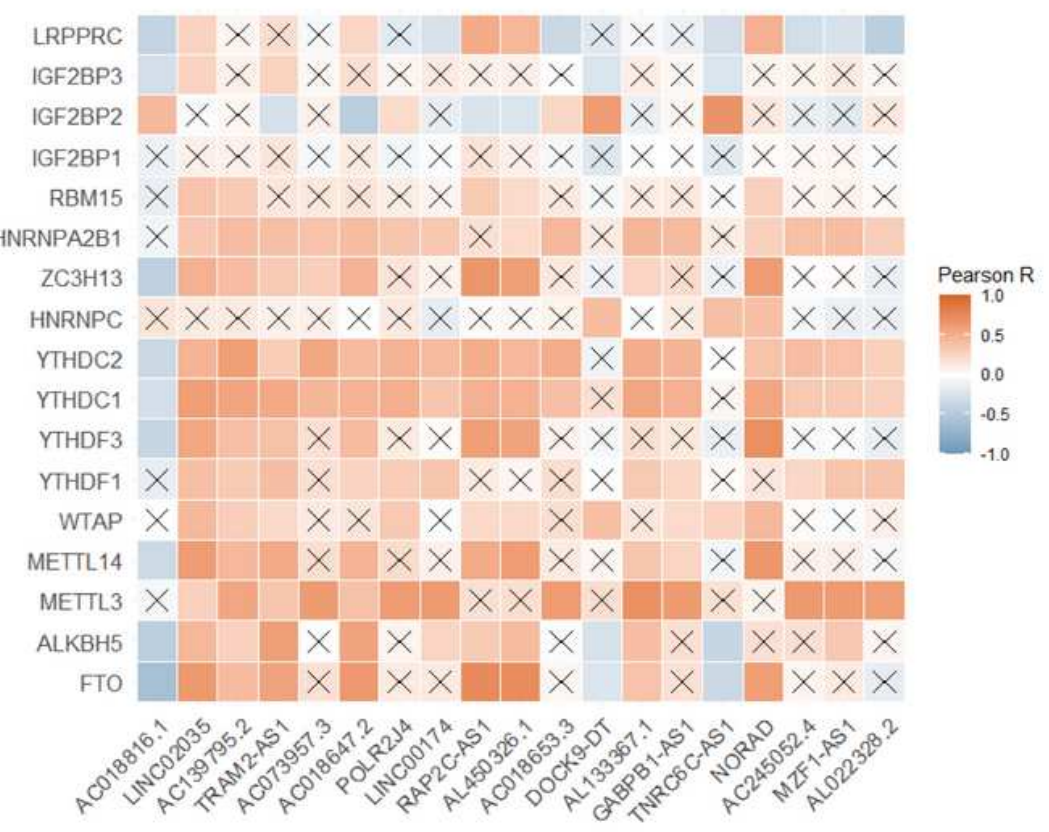

Figure 2

The profile of m6A -IncRNA regulators. A: Differential expression of 20 m6A RNA regulators; B: the correlations between m6A-related IncRNAs and m6A-related genes. ${ }^{*} p<0.05$, ${ }^{* *} p<0.01$, and ${ }^{* * *} p<0.001$. 

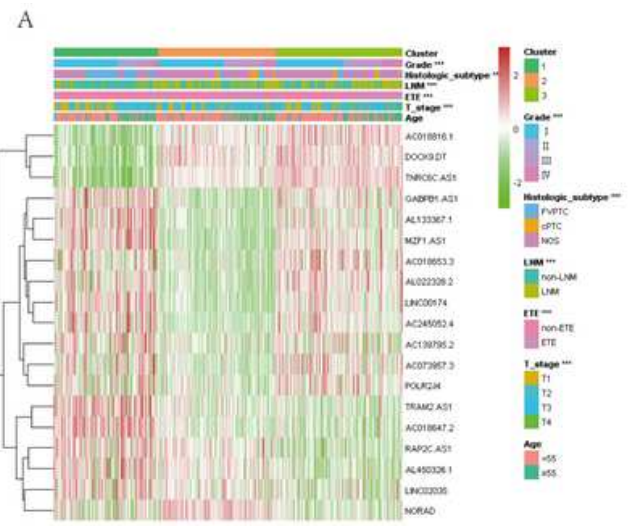

B

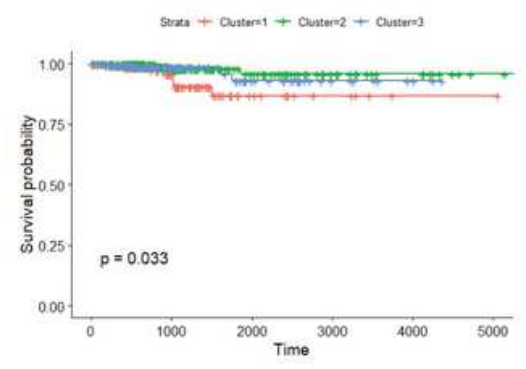

D

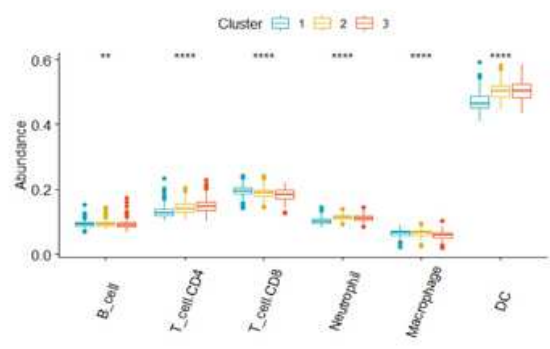

C
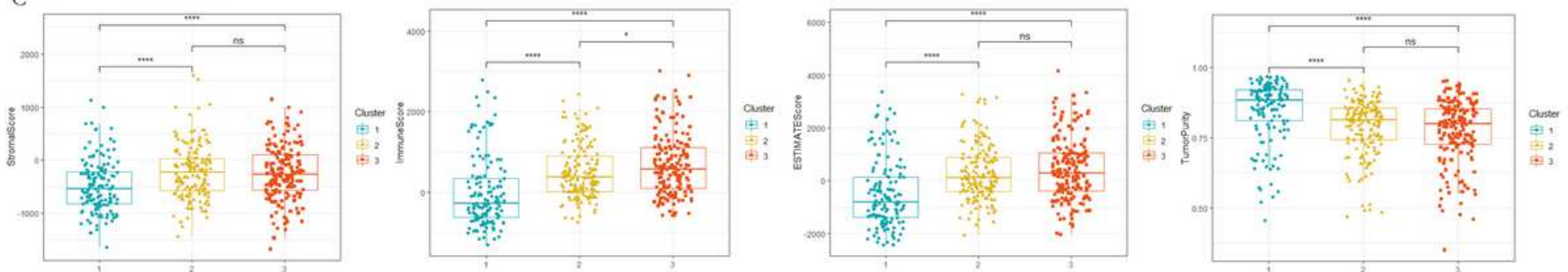

Figure 3

Prognosis and tumor immune microenvironment in consensus clustering. A: heatmap of cluster analysis clinicopathologic features; B: the OS of three subgroups; C-D: immunoscore and immune cell infiltration levels of cluster $1 / 2 / 3$ subtypes. OS: overall survival; LNM; lymph node metastasis; ETE: extrathyroidal extension. ${ }^{*} p<0.05,{ }^{*} \mathrm{p}<0.01,{ }^{* \star} \mathrm{p}<0.001$, and ns: no significance. 


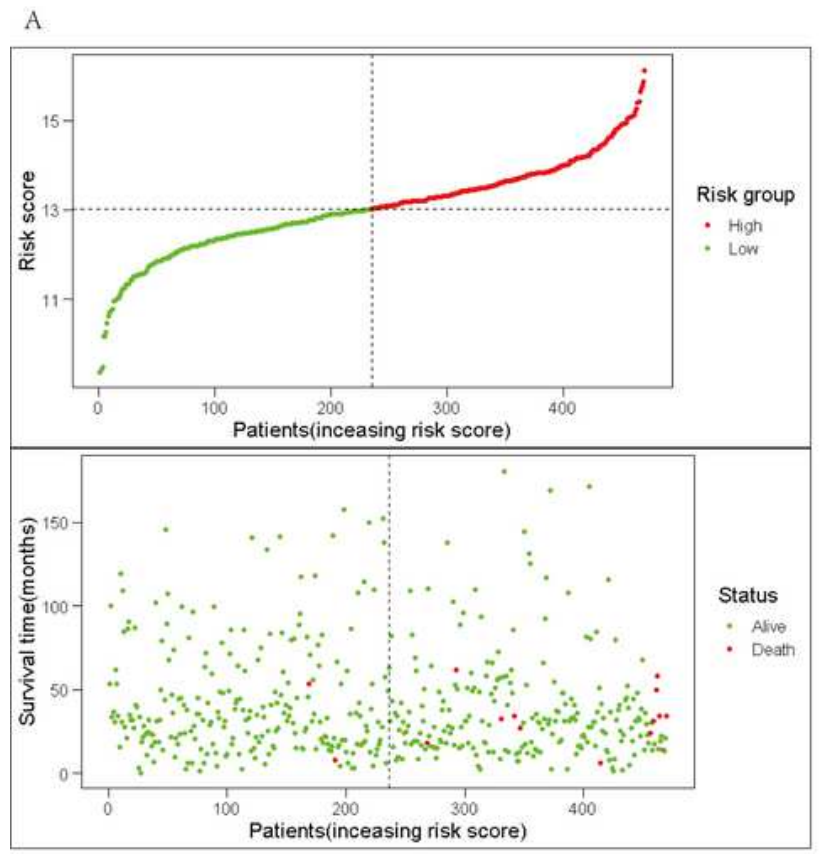

C

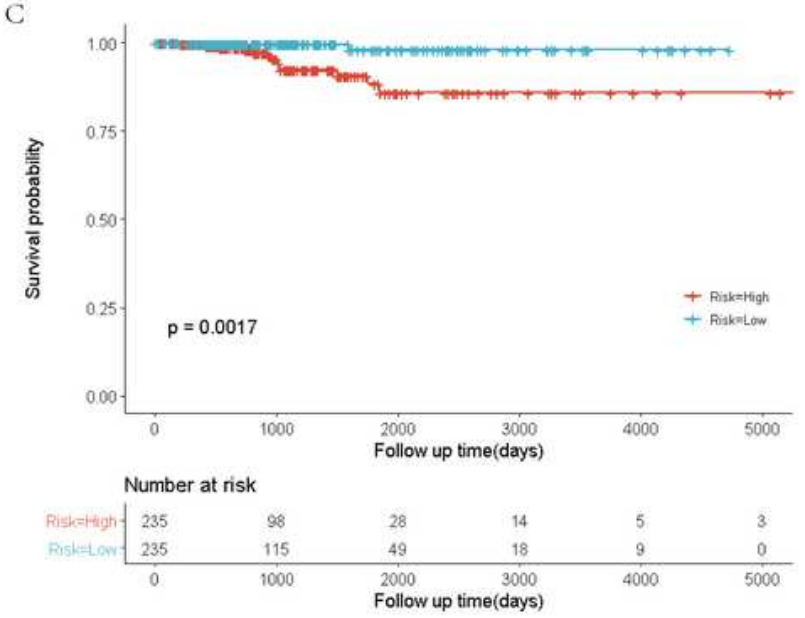

E

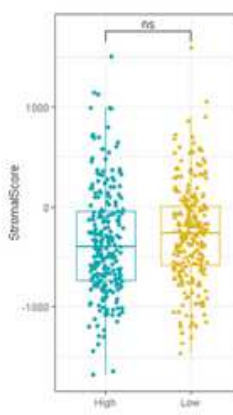

B

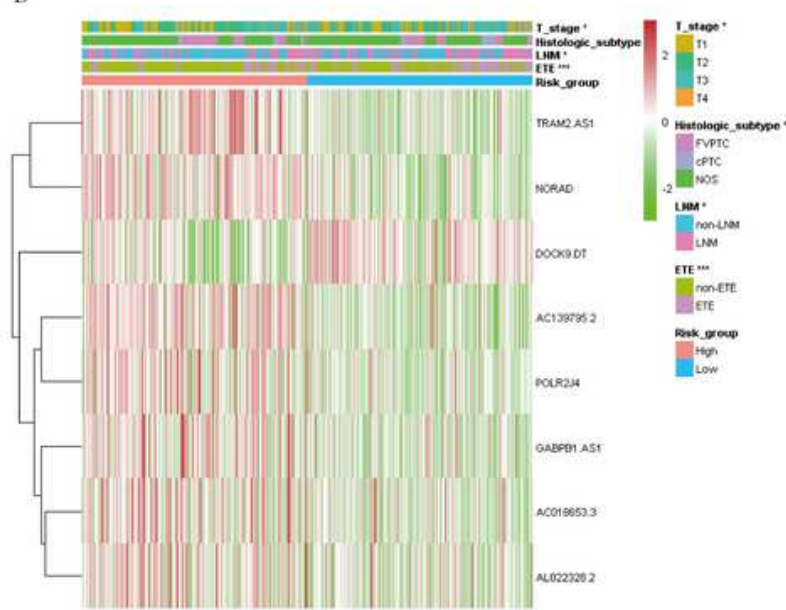

D
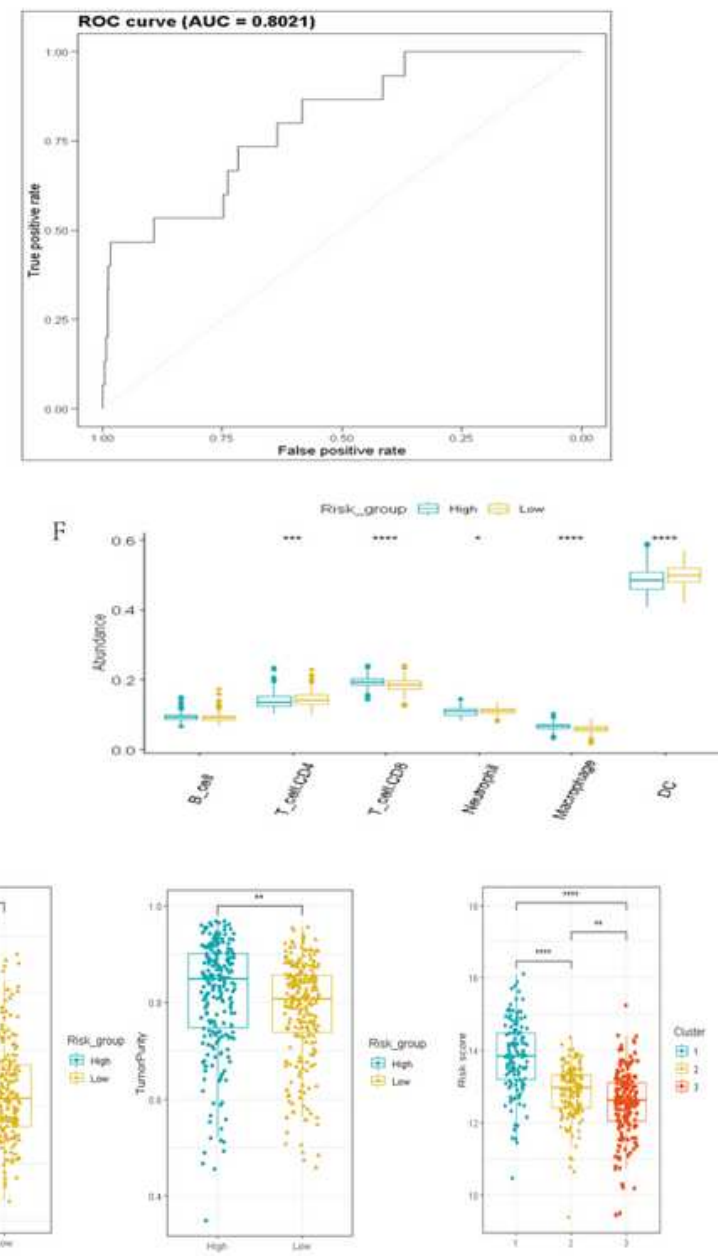

\section{Figure 4}

Construction and analysis of prognostic signatures of m6A-IncRNA regulators. A: the distributions of risk score, OS and OS status; $B$ : the heatmap analysis the clinicopathologic features and eight m6A-IncRNA between low- and high-risk groups; C: Kaplan-Meier curves of OS by risk score group; D: the AUC value of the risk score; E-F: the immune cell infiltration landscape in the risk score subgroups. OS: overall survival; 
AUC: the area of ROC curve. LNM; lymph node metastasis; ETE: extrathyroidal extension. ${ }^{*} p<0.05,{ }^{* *} p<$ $0.01, * * * p<0.001$, and ns: no significance.

A

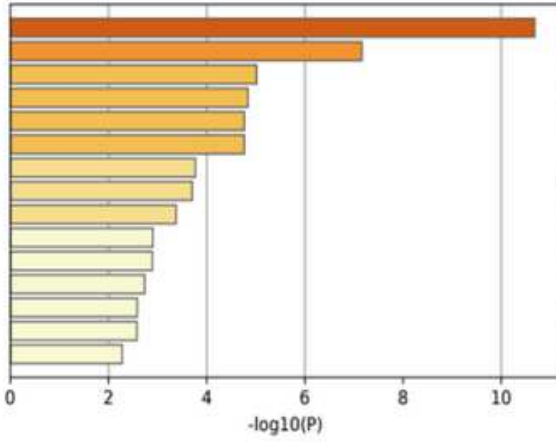

M5885: NABA MATRISOME ASSOCIATED

ko04918: Thyroid hormone synthesis

R-HSA-5683826: Surfactant metabolis

GO:0045055: regulated exocytosis

R-HSA-6785807: Interleukin-4 and Interleukin-13 signaling

R.HSA.425393: Transport of inorganic cations/anions and amino acids/oligopeptides

GO:0043627: response to estrogen

G0:0060395: SMAD protein signal transduction

GO:0022600: digestive system process

G0:0060562: epithelial tube morphogenesis

R-HSA-9018519: Estrogen-dependent gene expression

MS880: NABA ECM AFFILIATED

GO:0050708: regulation of protein secretion

GO:0071248: cellular response to metal ion

GO:0048871: multicellular organismal homeostasis
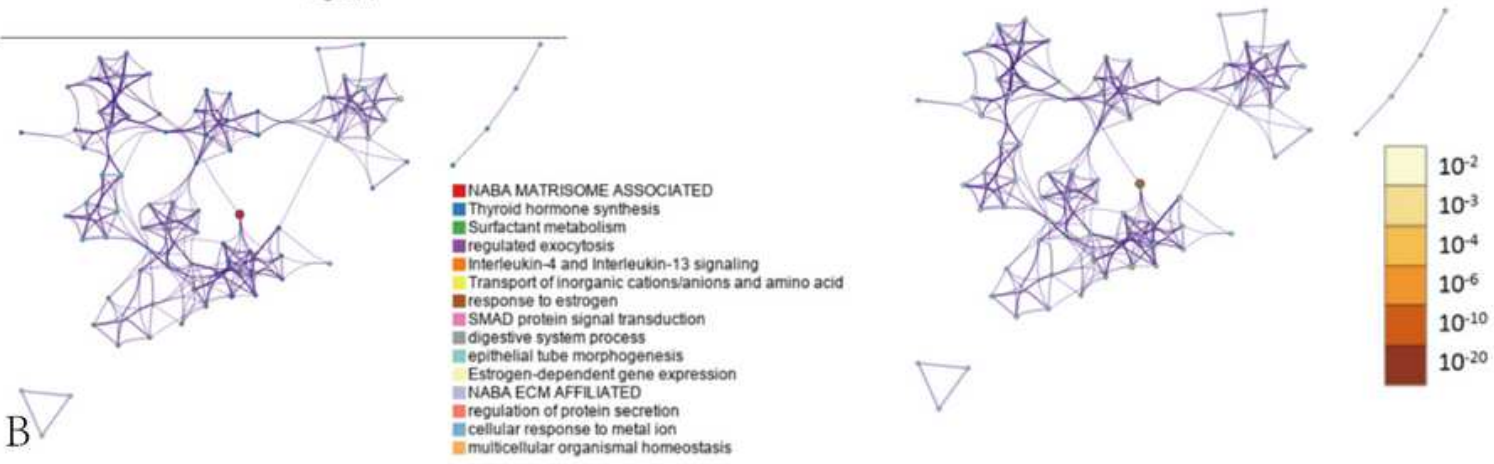

B

muliceellular organismal homeostasis
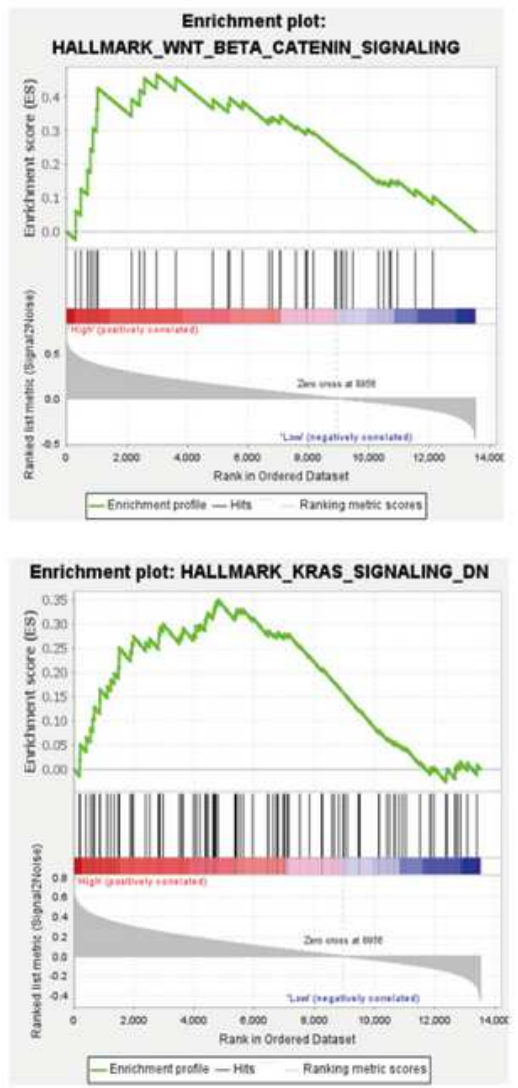

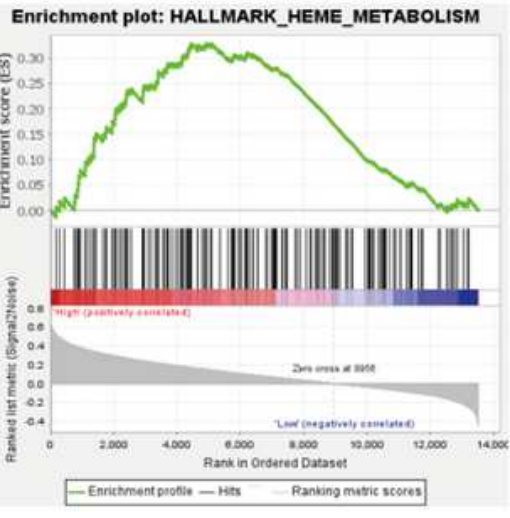

Enrichment plot: HALLMARK_BILE_ACID_METABOLISM
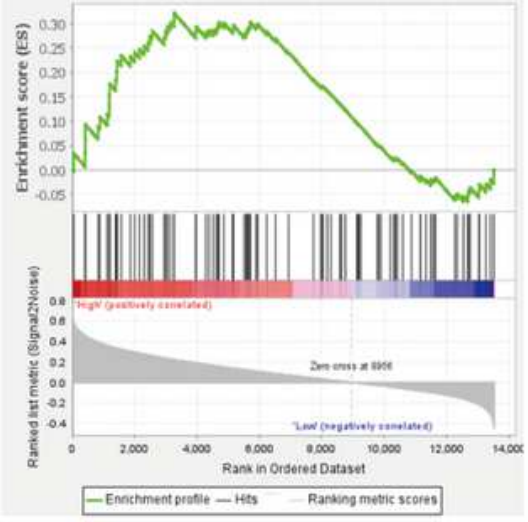
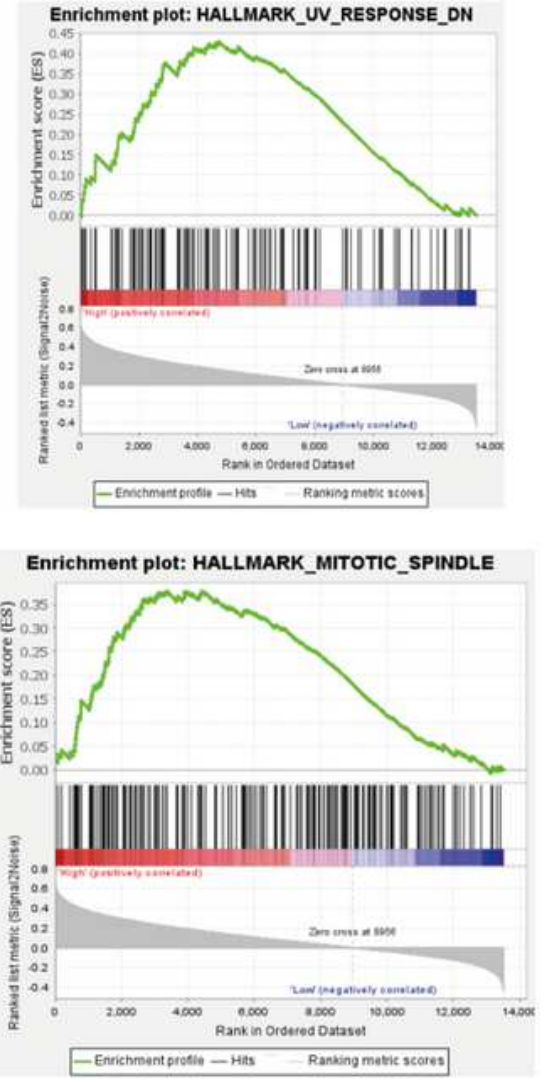

Figure 5

GSEA and Pathway and Functional enrichment analysis. A: KEGG pathway and GO function analysis; B: GSEA showed results in the high-risk group. KEGG: Kyoto Encyclopedia of Genes and Genomes; GO: Gene ontology; GSEA: Gene set enrichment analysis. 

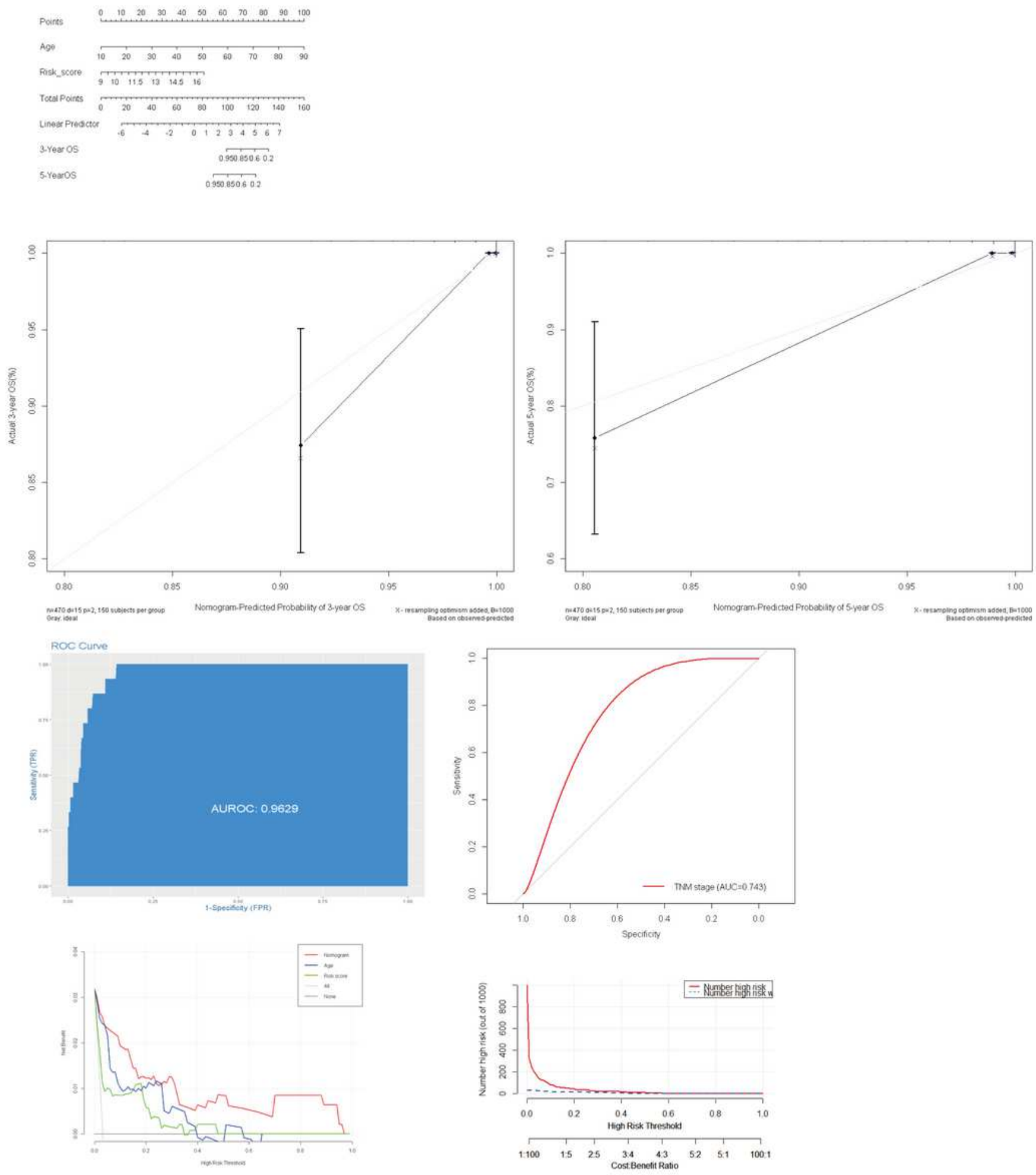

\section{Figure 6}

Construction and evaluation of prognostic nomogram model. A: Nomogram based on age and risk score for 3- and 5-year OS prediction; B: the calibration plots of nomogram model; C: the AUC value of the nomogram and AJCC TNM stage.D: decision curve analysis evaluated OS benefits. OS: overall survival. 
A
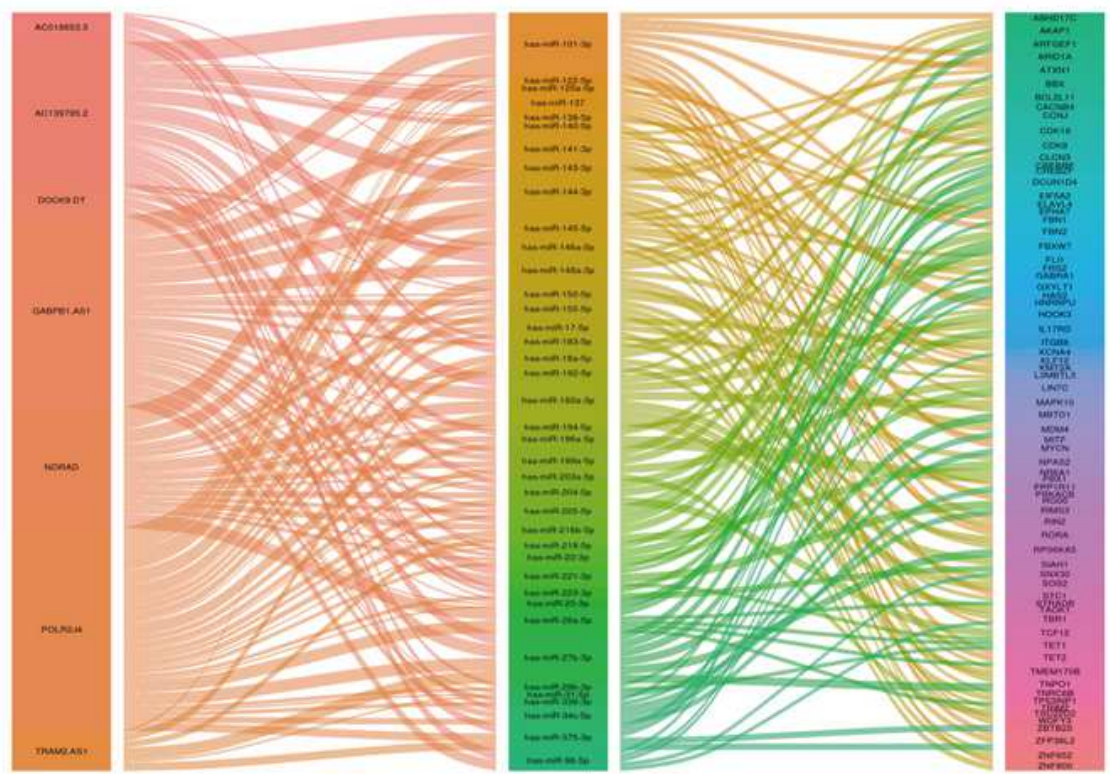

B

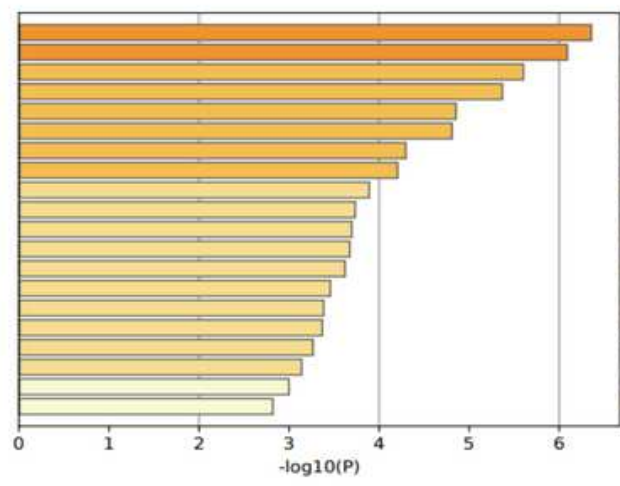

GO:0071385: cellular response to glucocorticoid stimulus GO:0001501: skeletal system development

GO:0045596: negative regulation of cell differentiation

60:0048511: hegatic process

GO:0030099: myeloid cell differentiation

hsa04910: Insulin signaling pathway

GO:0019827: stem cell population maintenance

R-HSA-2129379: Molecules associated with elastic fibres

hsa05202: Transcriptional misregulation in cancer

GO:0061013: regulation of mRNA catabolic process

GO:0001655: urogenital system development

R-HSA-1989781: PPARA activates gene expression

GO.0072698: protein localization to microtubule cytoskeleton

GO0.0051402: positive regulion of transter

M153: PID P75 NTR PATHWAY

GO:1901983. regulation of protein acetylation

R-HSA-5683057: MAPK family signaling cascades

$10(P$

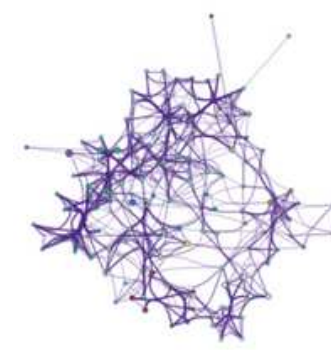

Conlular response bo glucoconcoid stmulus

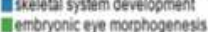
Whegative regulation of cell difterentation ilitingmic processs

myeloid cell difterentation

Insulin signaing pathway
in stem coll populason maintenance

- Molicules associaled with elastc fores

Transcriptonal mivegulaton in cancer

regulaton of mRNA catabolic process

Iurogenital spatem develooment

$$
\text { (2) }
$$
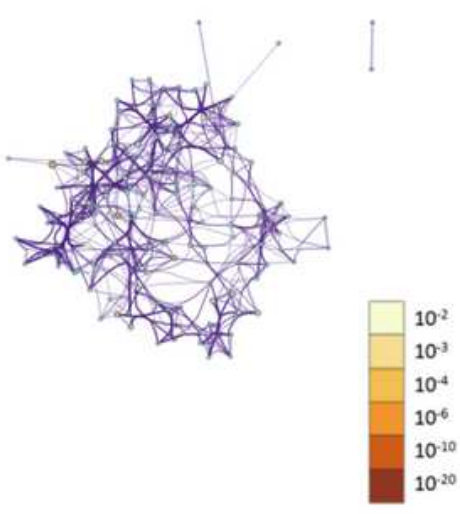

III protein localizaton to microtubule cyoskeleten

il positive reguaston of transterase activity

IIneuron apoptobc process

AVO guidance
PDO P75 NTR PATHWAY

iliegulation of protein acetyaton

\section{Figure 7}

The ceRNA network for PTC. 7A: ceRNA network was constracted by seven m6A-IncRNA, thirty-nine miRNAs and seventy-two mRNAs. 7B: functional and pathways enrichment analysis for seventy-two mRNAs. 

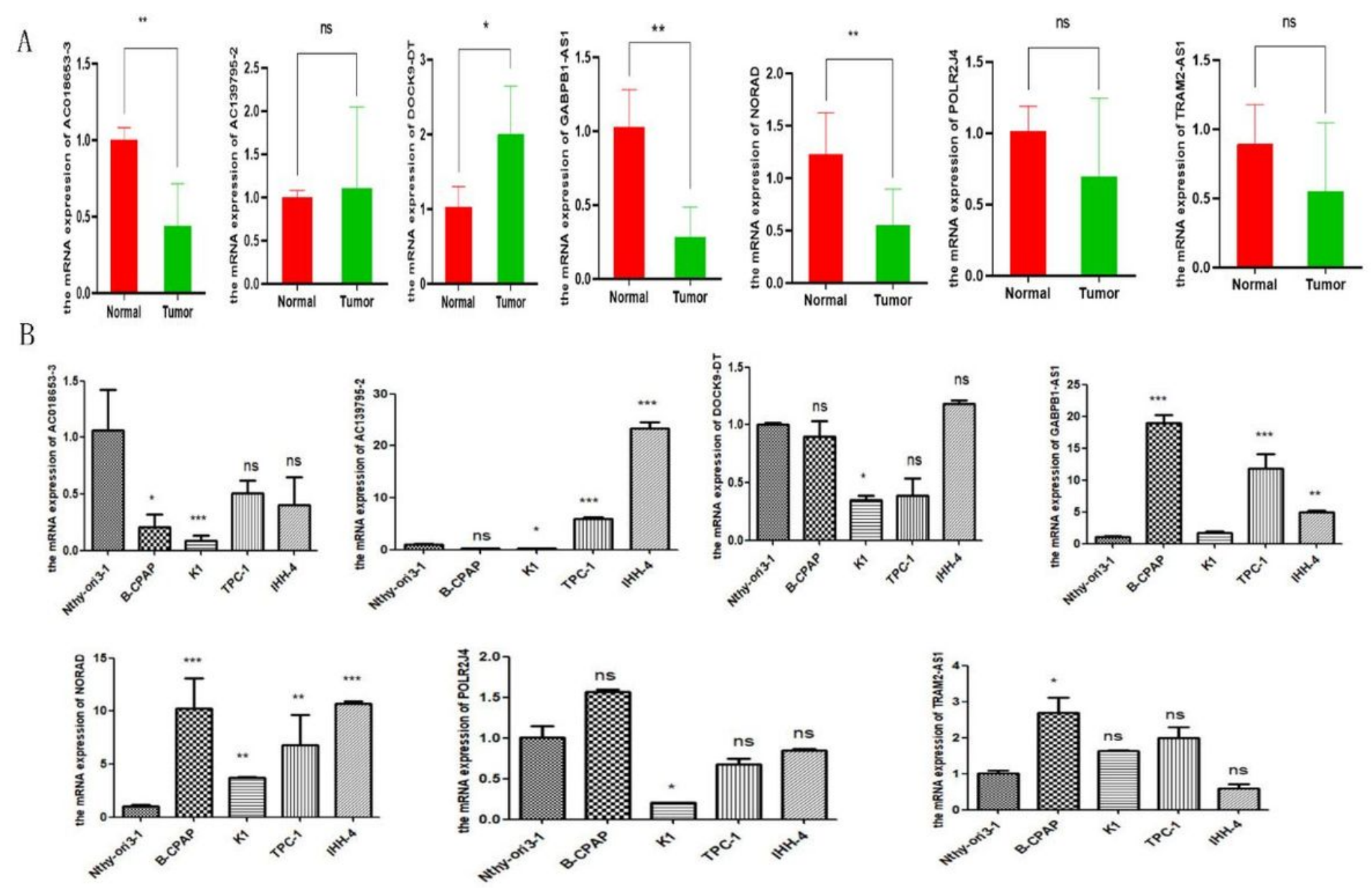

Figure 8

Validation the expression level of m5C-IncRNAs. A-B: the expression level of m5C-IncRNAs in 30 pairs PTC tissues and nine cells line. ${ }^{\star} p<0.05,{ }^{\star \star} p<0.01,{ }^{\star \star \star} p<0.001$, and ns: no significance.

\section{Supplementary Files}

This is a list of supplementary files associated with this preprint. Click to download.

- FigureS1.png

- Figures2.tif

- FigureS3.tif

- supplementarymaterial.docx 